\title{
Investigation of myxomycetes in Selcen Mountain (Turkey) and its
}

\section{close environs}

\author{
Murat ZÜMRE ${ }^{1 *}$, Hayri BABA ${ }^{1}$ and Mustafa SEVINDIK ${ }^{2 *}$ \\ ${ }^{1}$ Biology Dept. Faculty of Science and Literature, University of Hatay Mustafa Kemal, 31060 Hatay, Turkey \\ ${ }^{2}$ Department of Food Processing, Bahçe Vocational School, Osmaniye Korkut Ata University, Osmaniye, Turkey \\ Corresponding author: sevindik27@gmail.com
}

\begin{abstract}
In this study, myxomycetes, which have an important place in forest ecosystem, were determined. Myxomycetes act as saprophyte in the forest ecosystem. In this context, the myxomycet diversity of Selcen Mountain and its close environs was determined. This study has been made on the specimens which were obtained from 11 different station areas of Selcen Mountain and its close environs in 2011-2012. The samples were acquired from barks of trees, leaves and the materials of decayed trees. These materials were employed the Moist Chamber Culture and it was tried to develop myxomycetes sporophore. In addition myxomycetes were obtained from natural environment. As a result of field and laboratory studies 57 taxa belonging to 10 families and 21 genera were identified, and they were added to the Turkish Myxobiota.
\end{abstract}

Keywords: Myxomycetes, Diversity, Selcen Mountain.

\section{Özet}

Bu çalışma 2011-2012 yılları arasında, Selcen Dağı ve yakın çevresini kapsayan 11 ayrı istasyondan toplanan numuneler üzerinde yapılmıştır. Örnekler ağaç kabukları, yaprak, çürümüş veya çürümemiş bitkisel materyallerden elde edilmiştir. Toplanan numunelere Nem Odası Tekniği uygulanmış ve miksomiset sporoforları geliştirilmeye çalışılmıştır. Ayrıca doğal ortamında gelişmiş olan miksomisetlerde toplanmıştır. Elde edilen örneklerden arazi ve laboratuvar çalışmaları sonucu 10 familyaya ait 21 cins toplam 57 takson tespit edilmiştir. Belirlenen taksonlar Türkiye Miksobiotasına ilave edilmiştir.

Anahtar Kelimeler: Myxomycetes, Çeşitlilik, Selcen Dağı.

\section{Introduction}

Myxomycetes are small, relatively homogeneous group of eukaryotic organisms. Myxomycetes are multi- nucleate, lack of cell wall and free living organisms. The plasmodium a colorless or brightly colored vegetative body of myxomycetes that consists of multinucleate protoplasm lacking a membrane. According to last classification the myxomycetes classified in kingdom protista. Some groups of myxomycetes sometimes include lime in sporangium, out of the sporangium and sometimes both in sporangium and out of the sporangium which are important for taxonomy (Farr, 1981; Ergül and Akgül, 2011).

Some species of myxomycetes most widely distributed and the others keep their living in certain habitats. The myxomycetes are shown awareness on developed substrate and are sensitve to light, moisture, temperature and $\mathrm{pH}$. The myxomycetes live on bark of living trees, plant litter on the ground, aerial plant litter, standing dead wood or stumps, dead but still attached herbaceous plant parts 
such as old inflorescences, downed and decayed wood or bark, decaying fruit, herbivorous animal faces and animal bone. The myxomycetes feeding with other organism (bacteria, yeast, green algea) which are living in their habitat. The material which collected from the field bring to labrotory to developed myxomycetes in moist chamber culter (Gilbert ve Martin, 1933; Ergül et al., 2005a).

Like many microorganisms, myxomycets play an important role in the forest ecosystem. Results from studies carried out across different types of terrestrial ecosystems suggest that the species associated with coarse woody debris represent one of the main components of overall myxomycete diversity (Ing, 1994; Rufino and Cavalcanti, 2007; Takahashi and Harakon, 2012). Studies may help to determine the crucial biotic and abiotic factors determining species richness and diversity of myxomycetes in major ecosystems of the world (Novozhilov et al., 2017).

The number of myxomycetes are about 1017 in the world (Lado, 2019). The number of the myxomycetes in Turkey are 286 (Ergül et al., 2005b; Baba et al., 2013; Süerdem et al., 2015; Baba, 2015; Baba and Zümre., 2015; Alkan et al., 2016; Dülger et al., 2016; Ergül et al., 2016; Baba et al., 2016; Sesli et al., 2016; Baba and Arslan, 2017; Baba and Özyiğit, 2017; Baba and Er, 2018; Baba et al., 2018; Ocak and Konuk, 2018). Aim of this study identify myxomycetes of Selcen Mountain and its close environs.

\section{Materials and Methods}

Sampling Area

Yayladağ 1 is located in Hatay city in Akdeniz region. Antakya is located at the North of the town, Mediterranean sea is in the west, Syria at the east and South, Samandağ 1 is in the northwest and Altınözü is located at the northeast of the town. Yayladağ 1 is located between $35^{\circ} 48^{\prime}-36^{\circ} 04^{\prime}$ north latitude and $35^{\circ} 55^{\prime}-36^{\circ} 13^{\prime}$ east longitude. Map of the Study Area is below (Figure 1).

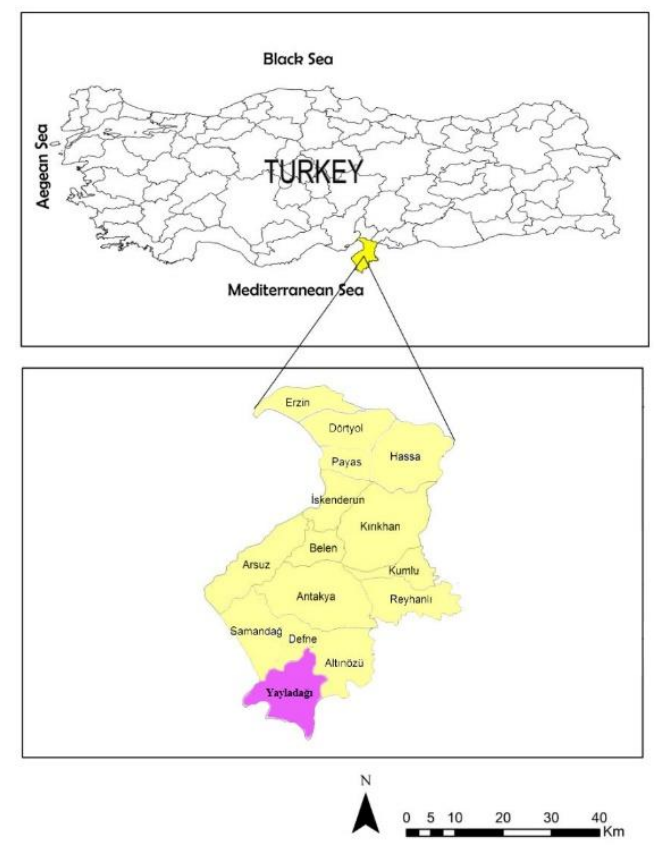

Figure 1. Map of the research area

In this study, 11 station was stated in Selcen Mountain and and its close environs in 2011-2012. In summer, autumn, winter and spring seasons was done field studies and sporophores were collected. 
Localities, date, altitude and coordinate of study area was shown in Table 1.

Table 1. Localities, date, altitude and coordinate of study area

\begin{tabular}{llccc}
\hline No & Localities & Date & Altitude (m) & Coordinate \\
\hline $\mathbf{1}$ & Eski Kapı Hill & 03.12 .2012 & $501 \mathrm{~m}$ & $35^{\circ} 54^{\prime} 39^{\prime \prime} \mathrm{N} ; 36^{\circ} 00^{\prime} 55^{\prime \prime} \mathrm{E}$ \\
$\mathbf{2}$ & Doruca Hill & 03.12 .2011 & $516 \mathrm{~m}$ & $35^{\circ} 54^{\prime} 13^{\prime \prime} \mathrm{N} ; 36^{\circ} 01^{\prime} 00^{\prime \prime} \mathrm{E}$ \\
$\mathbf{3}$ & Karpuzlu Hill & 17.12 .2011 & $489 \mathrm{~m}$ & $35^{\circ} 54^{\prime} 21^{\prime \prime} \mathrm{N} ; 36^{\circ} 01^{\prime} 41^{\prime \prime} \mathrm{E}$ \\
$\mathbf{4}$ & Salakçam Hill & 24.12 .2011 & $507 \mathrm{~m}$ & $35^{\circ} 54^{\prime} 45^{\prime \prime} \mathrm{N} ; 36^{\circ} 02^{\prime} 14^{\prime \prime} \mathrm{E}$ \\
$\mathbf{5}$ & Karpuzluk Hill & 05.02 .2012 & $476 \mathrm{~m}$ & $35^{\circ} 53^{\prime} 49^{\prime \prime} \mathrm{N} ; 36^{\circ} 01^{\prime} 41^{\prime \prime} \mathrm{E}$ \\
$\mathbf{6}$ & Dağardı Hill & 11.03 .2012 & $460 \mathrm{~m}$ & $35^{\circ} 54^{\prime} 21^{\prime \prime} \mathrm{N} ; 36^{\circ} 01^{\prime} 41^{\prime \prime} \mathrm{E}$ \\
$\mathbf{7}$ & Ölüali Hill & 18.03 .2012 & $486 \mathrm{~m}$ & $35^{\circ} 51^{\prime} 02^{\prime \prime} \mathrm{N} ; 36^{\circ} 02^{\prime} 29^{\prime \prime} \mathrm{E}$ \\
$\mathbf{8}$ & Habeştepe Hill & 25.03 .2012 & $540 \mathrm{~m}$ & $35^{\circ} 53^{\prime} 23^{\prime \prime} \mathrm{N} ; 36^{\circ} 03^{\prime} 45^{\prime \prime} \mathrm{E}$ \\
$\mathbf{9}$ & Köşker Hill & 28.04 .2012 & $585 \mathrm{~m}$ & $35^{\circ} 53^{\prime} 15^{\prime \prime} \mathrm{N} ; 36^{\circ} 06^{\prime} 36^{\prime \prime} \mathrm{E}$ \\
$\mathbf{1 0}$ & Ayvackk Farm & 28.04 .2012 & $589 \mathrm{~m}$ & $35^{\circ} 53^{\prime} 24^{\prime \prime} \mathrm{N} ; 36^{\circ} 04^{\prime} 50^{\prime \prime} \mathrm{E}$ \\
$\mathbf{1 1}$ & Alibey Farm & 28.04 .2012 & $569 \mathrm{~m}$ & $35^{\circ} 53^{\prime} 20^{\prime \prime} \mathrm{N} ; 36^{\circ} 05^{\prime} 28^{\prime \prime} \mathrm{E}$ \\
\hline
\end{tabular}

\section{Collecting samples and laboratory studies}

Myxomycetes sporophores were collected from barks, woods, organic material debris. It was then carefully placed in cardboard herbarium boxes. In addition, the fructification of myxomycetes were as obtained from the moist chamber culture in the laboratory. The cultures were moistened with distilled water. Moisturized sporophores were examined every day under a dissecting microscope (Baba et al., 2018).

\section{Identification of Samples}

For identification of samples was used stereo microscope and high definition light microscope. With the stereo microscope general structure, shape, color, macroscopic dimensions of fructification and, lime availability or color and shape of lime were analyzed. The capillitium, whether lack of the pseudokapillitium and columella, if available shape and dimensions, strands of capillitium ornementation, branch shape of the capillitium, situation of the columella free or not, features of the pseudocapillitium, shape, color, size and ornamentation of spores were studied in detail by light microscopy.

The identification of the samples was made by using different studies (Martin and Alexopoulos 1969; Farr 1976; Thind 1977; Farr 1981; Martin et al., 1983; Neubert et al., 1993; Neubert et al., 1995; Neubert et al., 2000; Stephenson and Stempen 1994; Alexopoulos et. al., 1996; Lado and Pando 1997; Ing 1999). The fungarium samples were stored in the laboratory of Department of Biology, Faculty of Arts and Science, Mustafa Kemal University.

\section{Results}

In this study 642 myxomycetes sporophores were obtained from 11 different station of Selcen Mountain and its close environs in 2011-2012. As a result of field and laboratory studies 57 taxa belonging to 10 families and 21 genera were identified. 


\section{Systematic classification}

Eukaryota

Protozoa

Amoebozoa

Myxomycetes

Ceratiomyxales

\section{Ceratiomyxaceae}

1. Ceratiomyxa fruticulosa (O.F. Müll.) T. Macbr., Karpuzluk Hill, on Pinus sp. L. wood, Zümre. 218.

Echinosteliales

\section{Echinosteliaceae}

2. Echinostelium minutum de Bary, Eski Kapı Hill, on P. brutia wood, Zümre. 1; Dağardı Farm, on P. brutia wood, Zümre. 297; Habeştepe Hill, on P. brutia wood, Zümre. 468; Köşker Hill, on cone stamps, Zümre. 540.

Liceales

\section{Cribrariaceae}

3. Cribraria cancellata (Batsch) Nann.-Bremek., Eski kap1 Hill, on P. brutia wood, Zümre. 1; Eski kap1 Hill, on P. brutia wood, Natural, Zümre. 64; Karpuzlu Hill, on P. brutia wood, Zümre. 101; Karpuzlu Hill, on P. brutia wood, Zümre. 164; Dağardı Farm, on P. brutia wood, Zümre.322; Ölüali Hill on P. brutia wood, Zümre. 376; Köşker Hill, on P. brutia wood, Zümre. 536; Ayvacık Farm, on P. brutia wood, Zümre. 569.

4. C. intricata Schrad., Karpuzlu Hill, on P. brutia wood, Zümre. 104; Dağardı Farm, on P. brutia wood, Zümre. 305.

5. C. macrocarpa Schrad., Eski kapı Hill, on P. brutia wood, Zümre. 58; Karpuzluk Hill, on P. brutia wood, Zümre. 230.

6. C. microcarpa (Schrad.) Pers., Eski kapı Hill, on P. brutia wood, Zümre. 53; Salakçam Hill, on P. brutia wood, Zümre. 164; Karpuzluk Hill, on P. brutia wood, Zümre. 240; Dağardı Farm, on P. brutia wood, Zümre. 295; Köşker Hill, on P. brutia wood, Zümre. 541; Ayvacık Farm, on P. brutia wood, Zümre. 565.

7. C. minutissima Schwein, Eski kapı Hill, on P. brutia wood, Zümre. 11; Köşker Hill, on P. brutia wood, Zümre. 533; Ayvacık Farm, on P. brutia wood, Zümre. 566; 569.

8. C. piriformis Schrad., Habeştepe Hill, on P. brutia wood, Zümre. 462.

9. C. vulgaris Schrad., Karpuzlu Hill, on P. brutia wood, Zümre. 105; Karpuzluk Hill, on P. brutia wood, Zümre. 226; Ölüali Hill, on P. brutia wood, Zümre. 378; Habeştepe Hill, on P. brutia wood, Zümre. 455; Habeştepe Hill, on P. brutia wood, Zümre. 462.

\section{Liceaceae}

10. Licea castanea G.Lister, Karpuzlu Hill, on P. brutia wood, Zümre. 101; Karpuzluk Hill, on P. brutia wood, Zümre. 226; Dağardı Farm, on P. brutia wood, Zümre. 295; Habeştepe Hill, on P. brutia wood, Zümre. 463; Köşker Hill, on Quercus sp., Zümre. 544.

11. L. kleistobolus G.W. Martin, Eski kapı Hill, on P. brutia wood, Zümre. 42; Habeştepe Hill, on $P$. brutia wood, Zümre. 471.

12. L. minima Fr., Eski kapı Hill, on P. brutia wood, Zümre. 31; Dağardı Farm, on P. brutia wood, Zümre. 322; Habeştepe Hill, on P. brutia wood, Zümre. 456; Köşker Hill, on P. brutia wood, Zümre. 538; Alibey Farm, on P. brutia wood, Zümre. 629. 


\section{Reticulariaceae}

13. Lycogala epidendrum (L.) Fr., Eski Kapı Hill, on P. brutia wood, Zümre. 1.

14. Reticularia lycoperdon Bull., Köşker Hill, on cortex of P. brutia, Zümre. 547.

Trichiales

\section{Arcyriaceae}

15. Arcyria affinis Rostaf., Dağardı Farm, on P. brutia wood, Zümre. 301.

16. A. cinerea (Bull.) Pers., Eski kapı Hill, on P. brutia wood, Zümre. 11; Karpuzluk Hill, on P. brutia wood, Zümre. 239; Dağardı Farm, on P. brutia wood, Zümre. 295; Ölüali Hill, on P. brutia wood, Zümre. 378; Habeştepe Hill, on P. brutia wood, Zümre. 451; Köşker Hill, on P. brutia wood, Zümre. 536; Alibey Farm, on P. brutia wood, Zümre. 601.

17. A. denudata (L.) Wettst., Karpuzluk Hill, on P. brutia wood, Zümre. 240; Habeştepe Hill, on Quercus sp. wood, Zümre. 464.

18. A. ferruginea Saut., Karpuzluk Hill, on P. brutia wood, Zümre. 240; Dağardı Farm, on P. brutia wood and bark, Zümre. 302; Habeştepe Hill, on Quercus sp. wood, Zümre. 469.

19. A. incarnata (Pers. ex J.F. Gmel.) Pers., Eski kapı Hill, on P. brutia wood and bark, Zümre. 4; Karpuzluk Hill, on P. brutia wood, Zümre. 232; Dağardı Farm, on P. brutia wood and bark, Zümre. 306; Köşker Hill, on P. brutia wood, Zümre. 561.

20. A. pomiformis (Leers) Rostaf., Eski kapı Hill, on $P$. brutia wood, Zümre. 31; Dağardı Farm, on $P$. brutia wood, Zümre. 296; Ölüali Hill, on P. brutia wood, Zümre. 380; Köşker Hill, on P. brutia wood, Zümre. 534; Alibey Farm, on P. brutia wood, Zümre. 597.

21. A. stipata (Schwein.) Lister, Ayvacık Farm, on P. brutia wood, Zümre. 564.

22. A. versicolor W. Phillips, Habeştepe Hill, on P. brutia wood, Zümre. 460.

\section{Trichiaceae}

23. Hemitrichia abietina (Wigand) G. Lister, Ölüali Hill, on P. brutia wood, Zümre. 380.

24. Trichia botrytis (J.F. Gmel.) Pers., Dağardı Farm, on Quercus sp. wood, Zümre. 303; Habeştepe Hill, on P. brutia wood, Zümre. 461; Köşker Hill, on Quercus sp. wood, Zümre. 537; Salakçam Hill, on P. brutia wood, Zümre. 174; Karpuzluk Hill, on P. brutia wood, Zümre. 225; Köşker Hill, on Quercus sp. wood, Zümre. 537.

25. T. crateriformis G.W. Martin, Karpuzluk Hill, on P. brutia Ten., wood, Zümre. 240; Dağard1 Farm, on P. brutia Ten., wood, Zümre. 322; Köşker Hill, on P. brutia Ten. bark, Zümre. 538.

26. T. decipiens (Pers.) T. Macbr., Dağardı Farm, on P. brutia wood, Zümre. 297.

27. T. erectra Rex., Eski kapı Hill, on P. brutia bark, Zümre. 19.

28. T. lutescens (Lister) Lister, Eski kapı Hill, on P. brutia wood, Zümre. 47; Salakçam Hill, on $P$. brutia wood, Zümre. 164; Habeştepe Hill, on P. brutia wood, Zümre. 459.

29. T. munda (Lister) Meyl. Eski kapı Hill, on P. brutia wood, Zümre. 53.

30. T. verrucosa Berk., Dağardı Farm, on P. brutia wood, Zümre. 302; Habeştepe Hill, on P. brutia wood, Zümre. 450.

Physarales

\section{Didymiaceae}

31. Diderma carneum Nann.- Bremek., Eski kap1 Hill, on P. brutia wood, Zümre. 62; Ölüali Hill, on P. brutia wood, Zümre. 367.

32. D. radiatum (L.) Morgan, Eski kapı Hill, on P. brutia wood, Zümre. 58; Ölüali Hill, on P. brutia wood, Zümre. 375.

33. Didymium bahiense Gottsb., Karpuzluk Hill, on P. brutia wood, Zümre. 244. 
34. D. difforme (Pers.) Gray, Habeştepe Hill, on P. brutia wood, Zümre. 451; Köşker Hill, on $P$. brutia wood, Zümre. 536.

35. D. melanospermum (Pers.) T. Macbr., Ölüali Hill, on P. brutia wood, Zümre. 370.

\section{Physaraceae}

36. Badhamia nitens Berk., Salakçam Hill, on P. brutia wood, Zümre. 164.

37. Physarum album (Bull.) Chevall., Eski kap1 Hill, on P. brutia wood, Zümre. 19; Karpuzluk Hill, on P. brutia wood, Zümre. 244.

38. P. flavicomum Berk., Eski kapı Hill, on P. brutia wood, Zümre. 10.

39. P. murinum Lister, Salakçam Hill, on P. brutia wood, Zümre. 167.

Stemonitales

\section{Stemonitidaceae}

40. Collaria lurida (Lister) Nann.-Bremek., Eski kap1 Hill, on P. brutia wood, Zümre. 11; Köşker Hill, on P. brutia wood, Zümre. 540.

41. Comatricha ellae Härk., Eski kapı Hill, on P. brutia wood, Zümre. 3; Karpuzlu Hill on P. brutia wood, Zümre. 117; Salakçam Hill, on P. brutia wood, Zümre. 168; Karpuzluk Hill, on P. brutia wood, Zümre. 239; Dağardı Farm, on P. brutia wood, Zümre. 296; Ölüali Hill, on P. brutia wood, Zümre. 386; Habeştepe Hill, on P. brutia wood, Zümre. 452; Köşker Hill, on P. brutia wood, Zümre. 535, Alibey Farm, on P. brutia wood, Zümre. 598.

42. C. elegans (Racib) G. Lister, Eski kapı Hill, on P. brutia wood, Zümre. 31, Ölüali Hill, on $P$. brutia wood, Zümre. 377.

43. C. nigra (Pers. ex J.F.Gmel.) J.Schröt., Eski kapı Hill, on Quercus sp. wood, Zümre. 5; Karpuzluk Hill, on P. brutia wood, Zümre. 234; Dağardı Farm, on P. brutia wood, Zümre. 298; Ölüali Hill, on $P$. brutia wood, Zümre. 383; Habeştepe Hill, on P. brutia wood, Zümre. 455; Alibey Farm, on P. brutia wood, Zümre. 599.

44. C. pulchella (C. Bab.) Rostaf., Eski kapı Hill, on P. brutia wood, Zümre. 16; Salakçam Hill, on Quercus sp. wood, Zümre. 160.

45. Enerthenema papillatum (Pers.) Rostaf., Eski kapı Hill, on P. brutia wood, Zümre. 28; Dağardı Farm, on P. brutia wood, Zümre. 297.

46. Lamproderma arcyrioides (Sommerf.) Rostaf., Karpuzluk Hill, on P. brutia wood, Zümre. 240.

47. L. laxum H. Neubert, Ölüali Hill, on P. brutia wood, Zümre. 370; Köşker Hill, on P. brutia wood, Zümre. 557.

48. Paradiacheopsis rigida (Brândza) Nann. -Bremek., Eski kap1 Hill, on P. brutia wood, Zümre. 23. 49. Stemonitis axifera (Bull.) T. Macbr., Eski kapı Hill, on P. brutia wood, Zümre. 14; Dağardı Farm, on P. brutia wood, Zümre. 298; Habeştepe Hill, on P. brutia wood, Zümre. 468; Köşker Hill, on P. brutia wood, Zümre. 539.

50. S. flavogenita E. Jahn, Karpuzluk Hill, on P. brutia wood and bark, Zümre. 226; Dağardı Farm, on P. brutia wood, Zümre. 318; Ölüali Hill, on P. brutia wood, Zümre. 373; Habeştepe Hill, on Quercus sp. bark, Zümre. 466; Köşker Hill, on P. brutia bark, Zümre. 544.

51. S. fusca Roth, Salakçam Hill, on P. brutia wood, Zümre. 180; Karpuzluk Hill, on P. brutia wood, Zümre. 296; Habeştepe Hill, on P. brutia wood, Zümre. 470; Ayvacık Farm, on P. brutia wood, Zümre. 570.

52. S. herbatica Peck, Alibey Farm, on P. brutia wood, Zümre. 594.

53. Stemonitopsis amoena (Nann.-Bremek.) Nann.-Bremek., Ölüali Hill, on P. brutia wood, Zümre. 382; Habeştepe Hill, on P. brutia wood, Zümre. 475. 
54. S. hyperopta (Meyl.) Nann.-Bremek., Eski kapı Hill, on P. brutia wood, Zümre. 22; Karpuzlu Hill, on P. brutia wood, Zümre. 135; Karpuzluk Hill, on P. brutia wood, Zümre. 235; Dağardı Farm, on P. brutia wood, Zümre. 295; Köşker Hill, on P. brutia wood, Zümre. 540.

55. S. reticulata (H.C. Gilbert) Nann.-Bremek.\& Y. Yamam., Ölüali Hill, on P. brutia wood, Zümre. 235; Dağardı Farm, on P. brutia wood, Zümre. 306; Alibey Farm, on P. brutia wood, Zümre. 595.

56. S. subcaespitosa (Peck) Nann.-Bremek., Köşker Hill, on P. brutia wood, Zümre. 534.

57. Symphytocarpus trechispora (Berk. ex Torrend) Nann.-Bremek., Dağardı Farm, on P. brutia wood, Zümre. 296.

\section{Discussion}

In this study, 57 taxa belonging to 10 families and 21 genera were identified in Selcen Mountain (Turkey) and its close environs. In our study, the distributions of families were determined as Ceratiomyxaceae 1, Echinosteliaceae 1, Cribrariaceae 7, Liceaceae 3, Reticulariaceae 2, Arcyriaceae 8, Trichiaceae 8, Didymiaceae 5, Physaraceae 4 and Stemonitidaceae 18 taxa respectively. In our study, 4 families (Cribrariaceae 7, Arcyriaceae 8, Trichiaceae 8 and Stemonitidaceae 18) constituted the majority of taxa. In this context, our study showed similarity with that of Yağız and Afyon (2005). In literature search samples of the myxomycetes mostly have been identified on the Gymnosperms rotted wood, leaves and debris (Martin ve Alexopoulos 1969; Ergül and Akgül, 2011; Baba et al., 2018). Members of Liceales, Trichiales and Stemonitales are usually grows in conifer forests (Martin and Alexopoulos 1969; Ergül and Akgül 2011; Baba et al., 2018). The majority of the samples that were identified have been deceted on the angiosperm debris.

Corticolous myxomycetes are found on decaying leaf litter, and still others on the bark of living trees and vines. Lignicolous myxomycetes are found on rotten wood and wood cortex. Foliicolous myxomycetes are found on leaves. Fimicolous myxomycetes are found on faces of the animals (Everhart and et. al., 2008). From the materials collected from the study area just from the cortex and rotten wood samples myxomycetes were obtained.

In addition, E. minutum, A. cinerea, A. denudata and S. fusca are cosmopolitan taxa (Stephenson and Stempen 1994). These species were also detected in our study area. E. minutum, A. pomiformis, A. cinerea, $C$. ellae and $C$. nigra have been identified in many areas of our study. In this context, it has been similar to the studies of many researchers (Ocak and Hasenekoğlu, 2003; Yağız and Afyon, 2007; Baba, 2015; Ergül et al., 2016).

\section{Conclusion}

In our study, myxomycetes were determined to be rotten in the forest ecosystem. In this context, myxomycetes were determined in Selcen Mountain (Turkey) and its close environs. As a result of field and laboratory studies 57 taxa belonging to 10 families and 21 genera were identified, and they were added to the Turkish Myxobiota. Identificated myxomycetes can contribute to determine of microhabitat located in the forest ecosystem.

\section{References}

Alexopoulos, C.J., Mims, C.W., Blackwell, M. (1996). Introductory Mycology, 4.th Edition, John Wiley and Sons Inc., New York.

Alkan, S., Eroğlu, G., Kaşı, G. (2016). A new myxomycete record from Turkey. Biological Diversity and Conservation 9(2): 128-130.

Baba, H. (2015). Investigation of Myxomycetes diversity on Kuseyr Mountain; Three new records in Hatay/Turkey. Fresen. Environ. Bull. 24(11c): 4077-4086. 
Baba, H., Arslan, Ç. (2017). Licea pescadorensis, A new Myxomycetes record for Turkey. Iğdır Univ. J. Inst. Sci. \& Tech., 7(4): 33-36.

Baba, H., Er, A. (2018). Craterium dictyosporum: A new record of Myxomycetes from Hatay, Turkey. Acta Biologica Turcica 31(1): 33-35

Baba, H., Gelen, M., Sevindik, M. (2018). Taxonomic investigation of myxomycetes in Altınözü, Turkey. Mycopath 16(1): 23-31

Baba, H., Gelen, M., Zümre, M. (2013). A new Myxomycetes record for Physarum Genus From Turkey. Biological Diversity and Conservation 6(3): 49-51.

Baba, H., Özyiğit, İ.İ. (2017). Three new rare Myxomycetes (Mycetozoa) records from Hatay, Turkey. Fresen. Environ. Bull. 26(8): 4907-4910.

Baba, H., Zümre, M. (2015). A new Myxomycetes record from Turkey. Journal of Biology, Agriculture and Healthcare 5(9): 14-16.

Baba, H., Zümre, M., Özyiğit, İ. (2016). A comparatıve biogeographical study of myxomycetes in four different habitats of eastern mediterranean part of Turkey, Fresen. Environ. Bull. 25(5): 1448-1459.

Dülger, B., Yıldız, I., Karabacak, E. (2016). A new myxomycetes record for the myxobiota of Turkey: Physarum melleum. International Journal of Botany Studies 1(4): 37-38.

Ergül, C.C., Akgül, H. (2011). Myxomycete diversity of Uludağ national park, Turkey. Mycotaxon 116:479.

Ergül, C.C., Akgül, H., Oran, R.B. (2016). New records of Mycetozoa taxa from Turkey. Oxidation Communications 39(2): 1615-1623.

Ergül, C.C., Dülger, B., Akgül, H. (2005a). Myxomycetes of Mezit stream valley of Turkey. Mycotaxon 92: 239-242

Ergül, C.C., Dülger, B., Oran, R.B., Akgül, H. (2005b). Myxomycetes of the western Black Sea Region of Turkey. Mycotaxon 93: 362

Everhart S. E., Keller H. W. (2008). L ife history strategies of corticolous myxomycetes: the life cycle, plasmodial types, fruiting bodies, and taxonomic orders. Fungal Diversity. 29: 1-16.

Farr, M. L. (1976). Flora Neotropica. Monograph No:16. N.Y. Bot. garden.

Farr, M. L. (1981). True Slime Molds. Wm. C. Brown Comp., p. 132, Dubuque Iowa.

Gilbert, H.C., Martin, G.W. (1933). Myxomycetes found on the bark of living trees. University of Iowa, Iowa Stud. Nat. Hist. 15 (3): 3-5.

Ing, B. (1999). The Myxomycetes of Britain and Ireland. The Richmond Publishing Co., Slough, England.

Ing, B. (1994). The phytosociology of myxomycetes. New Phytol. 126: 175-201.

Lado, C. (2019). An on line nomenclatural information system of Eumycetozoa. Real Jardín Botánico, CSIC. Madrid, Spain. Last updated March 01, 2019. http://www.nomen.eumycetozoa.com.

Lado, C., Pando, F. (1997). Flora Mycologica Iberica, Vol. 2. CSIC, p. 323, Madrid, Spain.

Martin, G. W., Alexopoulos, C. J. (1969). The Myxomycetes. University of Iowa press, p. 560, Iowa City.

Martin, G. W., Alexopoulos, C. J., Farr, M. L. (1983). The Genera of Myxomycetes. Univ. of Iowa Pres., p. 438, Iowa City.

Novozhilov, Y. K., Rollins, A. W., Schnittler, M. (2017). Ecology and distribution of myxomycetes. In Myxomycetes (pp. 253-297). Academic Press. 


\section{Eurasian Journal of Forest Science - Investigation of myxomycetes in Selcen Mountain \\ by Zïmre et al. 2019}

Neubert, H., Nowotny, W., Baumann, K. (1993). Die Myxomyceten (Band I). Karlheinz Baumann Verlag Gomaringen.

Neubert, H., Nowotny, W., Baumann, K. (1995). Die Myxomyceten (Band II). Karlheinz Baumann Verlag Gomaringen.

Neubert, H., Nowotny, W., Baumann, K., Marx, H. (2000). Die Myxomyceten (Band III). Karlheinz Baumann Verlag Gomaringen.

Ocak, İ., Hasenekoğlu, I. (2003a). Myxomycetes from Erzurum, Bayburt and Gümüşhane Provinces. Turk J Bot. 27: 223-226.

Ocak, İ., Konuk, M. (2018). Diversity and ecology of Myxomycetes from Kütahya and Konya (Turkey) with four new records. Mycobiology 46(3): 215-223.

Rufino, M.U.L., Cavalcanti, L.H. (2007). Alterations in the lignicolous myxomycete biota over two decades at the Dois Irmãos Ecologic State Reserve, Recife, Pernambuco, Brazil. Fungal Divers. 24: 159-171.

Sesli, E., Akata, I., Denchev, T.T., Denchev, C.M. (2016). Myxomycetes in Turkey - a checklist, Mycobiota 6: $1-20$.

Stephenson, S.L., Stempen, H. (1994). Myxomycetes: A Handbook of Slime Molds. Timber Press, Portland, Oregan, USA.

Süerdem T.B., Karabacak, E., Dülger, B. (2015). A new record of Diderma (Myxomycetes) from Turkey. Mycologia Iranica 2(2): 135 - 138.

Takahashi, K., Harakon, Y. (2012). Comparison of wood-inhabiting myxomycetes in subalpine and montane coniferous forests in the Yatsugatake Mountains of Central Japan. J. Plant Res. 125: 327-337.

Thind, K. S. (1977). The Myxomycetes of India. I.C.A.R., p.702, New Delhi

Yağız, D., Afyon, A. (2005). A study on the myxomycetes of Seydişehir (Konya) District. Afyon Kocatepe Üniversitesi Fen Bilimleri Dergisi 5: 55-60

Yağ1z, D., Afyon, A. (2007). The ecology and chorology of Myxomycetes in Turkey. Mycotaxon 101: 279-282.

Submitted: 21-07-2019 Accepted: 22-10-2019 